\title{
Lebesgue functions and Lebesgue constants in polynomial interpolation
}

Bayram Ali Ibrahimoglu*

\section{*Correspondence:} bibrahim@yildiz.edu.tr Department of Mathematical Engineering, Yıldız Technical University, Davutpasa Campus, Istanbul, 34210, Turkey

\begin{abstract}
The Lebesgue constant is a valuable numerical instrument for linear interpolation because it provides a measure of how close the interpolant of a function is to the best polynomial approximant of the function. Moreover, if the interpolant is computed by using the Lagrange basis, then the Lebesgue constant also expresses the conditioning of the interpolation problem. In addition, many publications have been devoted to the search for optimal interpolation points in the sense that these points lead to a minimal Lebesgue constant for the interpolation problems on the interval $[-1,1]$.

In Section 1 we introduce the univariate polynomial interpolation problem, for which we give two useful error formulas. The conditioning of polynomial interpolation is discussed in Section 2. A review of some results for the Lebesgue constants and the behavior of the Lebesgue functions in view of the optimal interpolation points is given in Section 3.
\end{abstract}

Keywords: polynomial interpolation; Lebesgue function; Lebesgue constant

\section{Introduction}

For $n \in \mathbb{N}$, let

$$
X=\left\{x_{j}: j=0,1, \ldots, n\right\}
$$

be a set of $n+1$ distinct interpolation points (or nodes) on the real interval $[-1,1]$ such that

$$
-1 \leq x_{0}<x_{1}<\cdots<x_{n} \leq 1
$$

Let the function $f$ belong to $C([-1,1])$. When approximating $f$ by an element from a finite-dimensional $\mathcal{V}_{n}=\operatorname{span}\left\{\phi_{0}, \ldots, \phi_{n}\right\}$ with $\phi_{i} \in C([-1,1])$ for $0 \leq i \leq n$, we know that there exists at least one element $p_{n}^{*} \in \mathcal{V}_{n}$ that is closest to $f$. When using the $\|\cdot\|_{\infty}$ norm, this element is the unique closest one if the $\phi_{0}, \ldots, \phi_{n}$ are a Chebyshev system. Since the computation of this element is more complicated than that of the interpolant

$$
\sum_{i=0}^{n} \alpha_{i} \phi_{i}\left(x_{j}\right)=f\left(x_{j}\right), \quad j=0, \ldots, n,-1 \leq x_{j} \leq 1,
$$

(c) 2016 Ibrahimoglu. This article is distributed under the terms of the Creative Commons Attribution 4.0 International License (http://creativecommons.org/licenses/by/4.0/), which permits unrestricted use, distribution, and reproduction in any medium, provided you give appropriate credit to the original author(s) and the source, provide a link to the Creative Commons license, and indicate if changes were made. 
there is an interest in interpolation points $x_{j}$ that make the interpolation error

$$
\left\|f(x)-\sum_{i=0}^{n} \alpha_{i} \phi_{i}(x)\right\|_{\infty}=\max _{x \in[-1,1]}\left|f(x)-\sum_{i=0}^{n} \alpha_{i} \phi_{i}(x)\right|
$$

as small as possible. In other words, there is an interest in using interpolating polynomials that are near-best approximants.

When $\phi_{i}(x)=x^{i}$ and $f$ is sufficiently differentiable, then for the interpolant

$$
p_{n}(x)=\sum_{i=0}^{n} \alpha_{i} x^{i}
$$

satisfying $p_{n}\left(x_{j}\right)=f\left(x_{j}\right), 0 \leq j \leq n$, the error $\left\|f-p_{n}\right\|_{\infty}$ is bounded by [1], pp.56-57

$$
\left\|f-p_{n}\right\|_{\infty} \leq \max _{x \in[-1,1]}\left(\frac{\left|f^{(n+1)}(x)\right|}{(n+1) !}\right) \max _{x \in[-1,1]} \prod_{j=0}^{n}\left|x-x_{j}\right| .
$$

In this study, we call this inequality the first error formula. It is well known that $\|(x-$ $\left.x_{0}\right) \cdots\left(x-x_{n}\right) \|_{\infty}$ is minimal on $[-1,1]$ if the $x_{j}$ are the zeroes of the $(n+1)$ th-degree Chebyshev polynomial $T_{n+1}(x)=\cos ((n+1) \arccos x)$.

The operator that associates with $f$ its interpolant $p_{n}$ is linear and given by

$$
P_{n}\left[x_{0}, \ldots, x_{n}\right]: C([-1,1]) \rightarrow \mathcal{V}_{n}: f(x) \rightarrow p_{n}(x)=\sum_{i=0}^{n} f\left(x_{i}\right) \ell_{i}(x),
$$

where the basic Lagrange polynomials

$$
\ell_{i}(x)=\prod_{j=0, i \neq j}^{n} \frac{x-x_{j}}{x_{i}-x_{j}}
$$

satisfy $\ell_{i}\left(x_{j}\right)=\delta_{i j}$. So another bound for the interpolation error is given by

$$
\left\|f-p_{n}\right\|_{\infty} \leq\left(1+\left\|P_{n}\right\|\right)\left\|f-p_{n}^{*}\right\|_{\infty}, \quad\left\|P_{n}\right\|=\max _{x \in[-1,1]} \sum_{i=0}^{n}\left|\ell_{i}(x)\right|,
$$

where $P_{n}:=P_{n}\left[x_{0}, \ldots, x_{n}\right]$ is the linear operator defined by $(4)$, and $p_{n}^{*}$ is the best uniform polynomial approximation to $f$.

\section{Lebesgue function and constant}

\subsection{Definition and properties}

Recall from (4) that

$$
p_{n}(x)=\sum_{i=0}^{n} f\left(x_{i}\right) \ell_{i}(x)
$$

where $p_{n}(x)$ is the Lagrange form for the polynomial that interpolates $f$ in the interpolation points $x_{0}, x_{1}, \ldots, x_{n}$ defined by (2), and the basic Lagrange polynomials $\ell_{i}(x)$ are defined by (5). 
For a fixed $n$ and given $x_{0}, \ldots, x_{n}$, the Lebesgue function is defined by

$$
L_{n}(x):=L_{n}\left(x_{0}, \ldots, x_{n} ; x\right)=\sum_{i=0}^{n}\left|\ell_{i}(x)\right|
$$

and the Lebesgue constant is defined by

$$
\Lambda_{n}:=\Lambda_{n}\left(x_{0}, \ldots, x_{n}\right)=\max _{-1 \leq x \leq 1} \sum_{i=0}^{n}\left|\ell_{i}(x)\right| .
$$

It is clear that both $L_{n}(x)$ and $\Lambda_{n}$ depend on the location of the interpolation points $x_{j}$ (and also on the degree $n$ ) but not on the function values $f\left(x_{i}\right)$. Note that the operator norm of $P_{n}$ defined by (4) is equal to the $\infty$-norm of its Lebesgue function:

$$
\left\|P_{n}\right\|_{\infty}=\Lambda_{n}=\max _{-1 \leq x \leq 1} L_{n}(x)
$$

Here and in the following, with the set $X$ defined by $(1)$, we sometimes write $L_{n}(X ; x):=$ $L_{n}\left(x_{0}, \ldots, x_{n} ; x\right)$ and $\Lambda_{n}(X):=\Lambda_{n}\left(x_{0}, \ldots, x_{n}\right)$ to simplify the notation.

The following present some basic properties of Lebesgue functions for Lagrange interpolation (see, e.g., $[2,3])$ :

(a) For any set $X$, with $n \geq 2, L_{n}(X ; x)$ is a piecewise polynomial satisfying $L_{n}(X ; x) \geq 1$ with equality only at the interpolation points $x_{j}, j=0, \ldots, n$.

(b) On each subinterval $\left(x_{j-1}, x_{j}\right)$ for $1 \leq j \leq n, L_{n}(X ; x)$ has precisely one local maximum, which is denoted by $\lambda_{j}(X)$. If the endpoints -1 and +1 are not interpolation points, that is, $-1<x_{0}$ and $x_{n}<1$, then there are two other subintervals and, thus, two other local maxima that are at -1 , and +1 . We denote the latter two local maxima by $\lambda_{0}(X)$ and $\lambda_{n+1}(X)$.

(c) The greatest and the smallest local maxima of $L_{n}(X ; x)$ are denoted correspondingly by $\mathcal{M}_{n}(X)$ and $m_{n}(X)$; we denote by $\delta_{n}(X)$ the maximum deviation among the local maxima $\delta_{n}(X)=\mathcal{M}_{n}(X)-m_{n}(X)$. We also denote the position of the Lebesgue constant (by taking one of the greatest local maxima) by $x^{*}(X)$ for the set of interpolation points $X$.

(d) The equality $L_{n}(X ; x)=L_{n}(X ;-x), x \in[-1,1]$, holds if and only if $x_{n-j}=-x_{j}, j=0, \ldots, n$.

(e) The Lebesgue constant is invariant under the linear transformation $t_{j}=\hat{a} x_{j}+\hat{b}, j=$ $0, \ldots, n(\hat{a} \neq 0)$. Interpolation sets that include the endpoints of the interval as interpolation points are called canonical interpolation sets. Let $\hat{X}$ denote a canonical interpolation set. In particular, we may construct a set $\hat{X}$ obtained from $X$ by mapping $\left[x_{0}, x_{n}\right]$ onto $[-1,1]$ by the unique linear transformation $t_{i}=\hat{a} x_{i}+\hat{b}, i=0, \ldots, n$, where $\hat{a}$ and $\hat{b}$ are determined by $-1=\hat{a} x_{0}+\hat{b}$ and $1=\hat{a} x_{n}+\hat{b}$. Here the set $\hat{X}$ is also called the canonicalization of the set $X$. We can see that the Lebesgue constant for $\hat{X}$ is [2, 4], pp.104-105

$$
\Lambda_{n}\left(t_{0}, \ldots, t_{n}\right)=\max _{x_{0} \leq x \leq x_{n}} L_{n}\left(x_{0}, \ldots, x_{n} ; x\right) \leq \max _{-1 \leq x \leq 1} L_{n}\left(x_{0}, \ldots, x_{n} ; x\right) .
$$

We use these properties in the sequel.

\subsection{Importance of Lebesgue constants}

One motivation for investigating the Lebesgue constant is that another upper bound for the interpolation error is given by

$$
\left\|f-p_{n}\right\|_{\infty} \leq\left(1+\Lambda_{n}\right)\left\|f-p_{n}^{*}\right\|_{\infty}
$$


where $p_{n}^{*}$ is the best polynomial approximation to $f$ on $[-1,1]$, and therefore $\Lambda_{n}$ quantifies how much larger the interpolation error $\left\|f-p_{n}\right\|_{\infty}$ is compared to the smallest possible error $\left\|f-p_{n}^{*}\right\|_{\infty}$ in the worst case. In this study, we call this inequality the Lebesgue inequality or the second error formula.

It is easy to show how to obtain this inequality. From the uniqueness of the interpolating polynomial we have $p_{n}(x)=\sum_{i=0}^{n} f\left(x_{i}\right) \ell_{i}(x)$ and $p_{n}^{*}(x)=\sum_{i=0}^{n} p_{n}^{*}\left(x_{i}\right) \ell_{i}(x)$. By subtracting $p_{n}(x)$ from $p_{n}^{*}(x)$ we get

$$
\begin{aligned}
\left|p_{n}^{*}(x)-p_{n}(x)\right| & =\left|\sum_{i=0}^{n} p_{n}^{*}\left(x_{i}\right) \ell_{i}(x)-\sum_{i=0}^{n} f\left(x_{i}\right) \ell_{i}(x)\right| \\
& =\left|\sum_{i=0}^{n}\left(p_{n}^{*}\left(x_{i}\right)-f\left(x_{i}\right)\right) \ell_{i}(x)\right| \\
& \leq \sum_{i=0}^{n}\left|\ell_{i}(x)\right| \max _{i=0, \ldots, n}\left|p_{n}^{*}\left(x_{i}\right)-f\left(x_{i}\right)\right| .
\end{aligned}
$$

From this it follows that (due to $\left.L_{n}(x)=\sum_{i=0}^{n}\left|\ell_{i}(x)\right|\right)$

$$
\left\|p_{n}^{*}-p_{n}\right\|_{\infty} \leq \Lambda_{n}\left\|f-p_{n}^{*}\right\|_{\infty}
$$

Finally, we have

$$
\begin{aligned}
\left\|f-p_{n}\right\|_{\infty} & =\left\|f-p_{n}^{*}+p_{n}^{*}-p_{n}\right\|_{\infty} \\
& \leq\left\|f-p_{n}^{*}\right\|_{\infty}+\left\|p_{n}^{*}-p_{n}\right\|_{\infty} \\
& \leq\left\|f-p_{n}^{*}\right\|_{\infty}+\Lambda_{n}\left\|f-p_{n}^{*}\right\|_{\infty} \\
& =\left(1+\Lambda_{n}\right)\left\|f-p_{n}^{*}\right\|_{\infty} .
\end{aligned}
$$

As a simple consequence of this inequality, it is obvious that $p_{n} \rightarrow f$ as the factor $\Lambda_{n} \| f-$ $p_{n}^{*} \|_{\infty} \rightarrow 0$. Namely, the Lebesgue inequality indicates that for the interpolation of a fixed function $f$ on $[-1,1]$, convergence can be expected only if $f$ is smooth enough such that $\left\|f-p_{n}^{*}\right\|_{\infty}$ decreases, as $n \rightarrow \infty$, faster than $\Lambda_{n}$ increases.

Another motivation for investigating the Lebesgue constant is that the Lebesgue constant also expresses the conditioning of the polynomial interpolation problem in the Lagrange basis. Let $\tilde{p}_{n}(x)$ denote the polynomial interpolant of degree $n$ for the perturbed function $\tilde{f}$ in the same interpolation points:

$$
\tilde{p}_{n}(x)=\sum_{i=0}^{n} \tilde{f}\left(x_{i}\right) \ell_{i}(x)
$$

Since $\left\|p_{n}\right\|_{\infty} \geq \max _{i=0, \ldots, n}\left|f\left(x_{i}\right)\right|$, we have

$$
\begin{aligned}
\frac{\left\|p_{n}-\tilde{p}_{n}\right\|_{\infty}}{\left\|p_{n}\right\|_{\infty}} & \leq \frac{\max _{x \in[-1,1]} \sum_{i=0}^{n}\left|f\left(x_{i}\right)-\tilde{f}\left(x_{i}\right)\right|\left|\ell_{i}(x)\right|}{\max _{i=0, \ldots, n}\left|f\left(x_{i}\right)\right|} \\
& \leq \Lambda_{n}\left(x_{0}, \ldots, x_{n}\right) \frac{\max _{i=0, \ldots, n}\left|f\left(x_{i}\right)-\tilde{f}\left(x_{i}\right)\right|}{\max _{i=0, \ldots, n}\left|f\left(x_{i}\right)\right|}
\end{aligned}
$$


This indicates that if we are able to choose interpolation points such that $\Lambda_{n}$ is small, then we can find the Lagrange interpolant that is less sensitive to errors in the function values. For this reason, numerical interpolation in floating-point arithmetic will generally be useless, even for smooth functions $f$, whenever the Lebesgue constant $\Lambda_{n}$ is larger than the inverse of the machine precision, which is typically about $10^{16}$.

\section{Some specific sets of interpolation points}

This section gives a summary of some results for particular sets of interpolation points for which the behavior of the Lebesgue function has been well investigated.

\subsection{Equidistant nodes}

There are many studies on the behavior of the Lebesgue function corresponding to the set of equidistant points although this set is a bad choice for polynomial interpolation owing to the Runge phenomenon.

For the set of equidistant points

$$
E=\left\{x_{j}=-1+\frac{2 j}{n}, j=0,1, \ldots, n\right\}
$$

the Lebesgue constant $\Lambda_{n}(E)$ grows exponentially with the asymptotic estimate [5, 6]

$$
\Lambda_{n}(E) \simeq \frac{2^{n+1}}{e n(\log n+\gamma)}, \quad n \rightarrow \infty
$$

where

$$
\gamma=\lim _{n \rightarrow \infty}\left(\sum_{i=1}^{n} \frac{1}{i}-\log n\right)=0.577 \ldots
$$

is Euler's constant (or the Euler-Mascheroni constant). Also, an asymptotic expansion that improves (9) (with unknown explicit general formula for the series coefficients) is found in [7].

For $\Lambda_{n}(E)$, the upper and lower bounds

$$
\frac{2^{n-2}}{n^{2}}<\Lambda_{n}(E)<\frac{2^{n+3}}{n}, \quad n \geq 1,
$$

have been suggested [8]. In [5], an upper bound is given for the smallest local maxima $m_{n}(E)$ :

$$
m_{n}(E)<\frac{2}{\pi}(\log (n+2)+\log 2+\gamma) .
$$

From (10) together with (11) it follows that $\Lambda_{n}(E)$ and $m_{n}(E)$ are of different orders of magnitude, and hence the maximum deviation $\delta_{n}(E)$ of the local maxima tends to infinity exponentially fast. As Figure 1 (left) illustrates, the Lebesgue function $L_{n}(E ; x)$ has wild oscillations near the endpoints, like the Runge phenomenon in the error curve. The local maxima of $L_{n}(E ; x)$ decrease strictly from the outside toward the midpoint of the interval $[-1,1][9]$. This behavior suggests that the location of the Lebesgue constant is in the first 

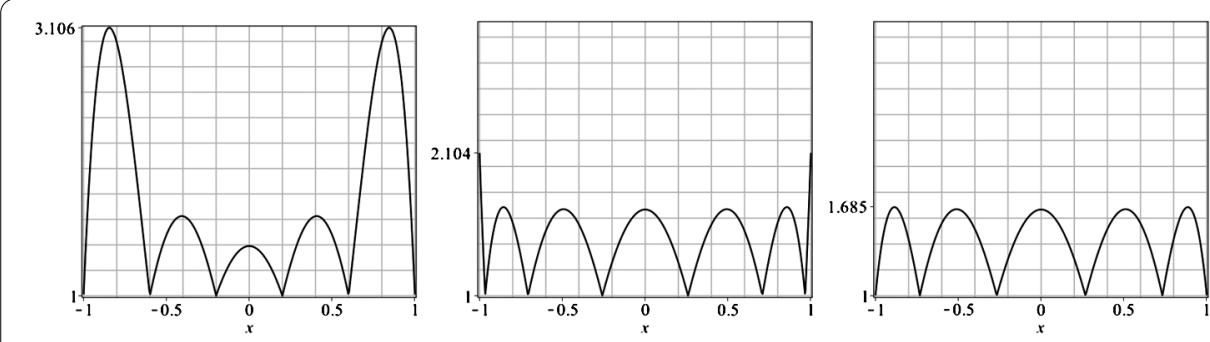

Figure 1 Graphs of $L_{5}(E ; x)$ (left), $L_{5}(T ; x)$ (center), $L_{5}(\hat{T} ; x)$ (right).

Figure 26 Chebyshev ( 0 ) and extended Chebyshev $(\bullet)$ nodes.

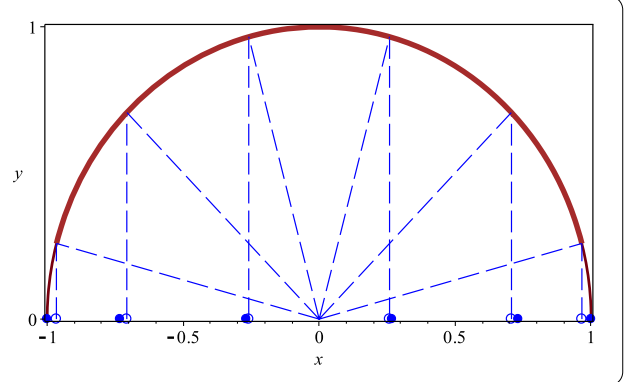

subinterval (or due to symmetry in the last subinterval). Numerical observation shows that the location of the Lebesgue constant occurs near the midpoint of the last (or first) subinterval, that is, $x^{*}(E) \approx(n-1) / n$ for the interval $[-1,1]$.

From the Lebesgue inequality (6) we know that equidistant points with this very fast growth of the Lebesgue constant give very poor approximations as $n$ increases. Indeed, numerical experiments show that for degree $n \geq 65$, the Lebesgue constant $\Lambda_{n}(E)$ reaches the inverse of the machine precision.

\subsection{Chebyshev nodes of the first kind}

The literature describes numerous investigations for the behavior of the Lebesgue function corresponding to the set of Chebyshev nodes. They are a very good choice of points for polynomial interpolation, and as it was indicated in Section 1, they give the smallest upper bound for the interpolation error of polynomial interpolation. As illustrated in Figure 2, they are obtained by projecting equally spaced points on a semicircle down to the unit interval $[-1,1]$; see the explicit formula (12).

The set of Chebyshev points

$$
T=\left\{x_{j}=-\cos \left(\frac{\pi}{2} \frac{(2 j+1)}{(n+1)}\right), j=0,1, \ldots, n\right\}
$$

is distributed more densely toward the endpoints of the interval $[-1,1]$, as illustrated in Figure 3 for $n=32$.

The Lebesgue constant $\Lambda_{n}(T)$ for polynomial interpolation grows logarithmically with the asymptotic expression $[10]$

$$
\Lambda_{n}(T)=\frac{2}{\pi}\left(\log (n+1)+\gamma+\log \frac{8}{\pi}\right)+\alpha_{n}, \quad 0<\alpha_{n}<\frac{\pi}{72(n+1)^{2}}, n \geq 1,
$$




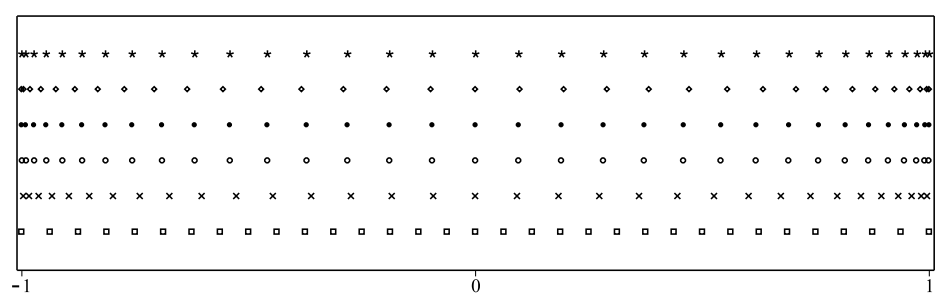

Figure 3 Graphs of sets of 33 nodes; $(*) \breve{T},(\diamond) \bar{U},(\bullet) \hat{T},(\circ) T,(x) U,(\square) E$ from top to bottom.

from which the upper and lower bounds

$$
\frac{2}{\pi} \log (n+1)+0.9625 \ldots<\Lambda_{n}(T)<\frac{2}{\pi} \log (n+1)+0.9734 \ldots, \quad n \geq 1
$$

can be deduced.

For $\Lambda_{n}(T)$, an asymptotic series expansion, which is valid for all finite $n$, is given by $[10-12]$

$$
\Lambda_{n}(T)=\frac{2}{\pi}\left(\log (n+1)+\gamma+\log \frac{8}{\pi}\right)+\sum_{v=1}^{\infty} \frac{\mathcal{A}_{v}}{(n+1)^{2 v}}, \quad n \geq 1,
$$

where the coefficients $\mathcal{A}_{v}$ have alternating signs and can be calculated as

$$
\mathcal{A}_{v}=(-1)^{v-1} \frac{4}{\pi} \frac{1-2^{1-2 v}}{(2 \pi)^{2 v}}(2 v-1) ! \zeta(2 v)\left(1+\sum_{j=v+1}^{\infty} \frac{\zeta(2 j)}{(2)^{2 j-1}}\left(\begin{array}{c}
2 j-1 \\
2 v-1
\end{array}\right)\right),
$$

where

$$
\zeta(s)=\sum_{k=1}^{\infty} \frac{1}{k^{s}}
$$

is the Riemann zeta function.

Using the little-o notation defined by $\varepsilon(n)=o(e(n))$ when $\varepsilon(n) / e(n) \rightarrow 0, n \rightarrow \infty$, Brutman showed [13] that

$$
m_{n}(T)=\Lambda_{\left\lfloor\frac{n}{2}\right\rfloor}(T)+o(1), \quad n \geq 2,
$$

from which the lower bound

$$
\frac{2}{\pi} \log (n+1)+\underbrace{\frac{2}{\pi}\left(\log \frac{4}{\pi}+\gamma\right)}_{0.521251 \ldots}<m_{n}(T)
$$

is obtained. Later, this bound was improved [14] as follows:

$$
\frac{2}{\pi}\left(\log (n+1)+\log \frac{4}{\pi}+\gamma\right)+\frac{\pi}{18(n+1)^{2}}-\frac{49 \pi^{3}}{10,800(n+1)^{4}}<m_{n}(T) .
$$


A comparison of (13) and (14) shows that the deviation between any two local maxima of the Lebesgue function $L_{n}(T ; x)$ does not exceed 0.4522 . This result was improved in [10] to

$$
\delta_{n}(T)=\mathcal{M}_{n}(T)-m_{n}(T) \leq \frac{2}{\pi} \log 2=0.44127
$$

As Figure 1 (center) suggests, the local maxima of $L_{n}(T ; x)$ are decreasing strictly from the outside toward the midpoint of the interval $[-1,1]$, which was proven in [13]. The figure also shows that the location of the Lebesgue constant occurs at \pm 1 , that is, $x^{*}(T)= \pm 1[15$, $16]$.

We know from the first error formula (3) that the Chebyshev points are a good choice for polynomial interpolation. Now, this slow growth of the Lebesgue constant confirms that they are also a good choice for the second error formula (6), which becomes

$$
\left\|f-p_{n}\right\|_{\infty} \leq\left(\frac{2}{\pi} \log (n+1)+2\right)\left\|f-p_{n}^{*}\right\|_{\infty}
$$

for the Chebyshev nodes. For example, for $n=100$, the interpolation error based on the Chebyshev points is

$$
\left\|f-p_{n}\right\|_{\infty} \leq\left(4.9 \times 10^{0}\right)\left\|f-p_{n}^{*}\right\|_{\infty}
$$

that is, even in the worst case, the interpolation error $\left\|f-p_{n}\right\|_{\infty}$ is only 4.9 times larger than the smallest possible error. For comparison, if we choose equidistant points for the same degree, then the upper bound for the interpolation error is

$$
\left\|f-p_{n}\right\|_{\infty} \leq\left(1.8 \times 10^{27}\right)\left\|f-p_{n}^{*}\right\|_{\infty} .
$$

\subsection{Extended Chebyshev nodes}

The extended Chebyshev nodes $\hat{T}$ are defined by

$$
\hat{T}=\left\{x_{j}=-\cos \left(\frac{\pi}{2} \frac{(2 j+1)}{(n+1)}\right) / \cos \left(\frac{\pi}{2(n+1)}\right), j=0,1, \ldots, n\right\},
$$

where the division by $\cos (\pi / 2(n+1))$ guarantees that $x_{0}=-1$ and $x_{n}=1$, and the set $\hat{T}$ is obtained from the set $T$ by the linear transformation that maps $\left[x_{0}, x_{n}\right]$ onto $[-1,1]$. Therefore, the set $\hat{T}$ is the canonicalization of the Chebyshev set $T$ (see Figures 2 and 3).

From the monotonicity result for the local maxima of $L_{n}(T ; x)$ and property (e) given in Section $2.1[2,16]$,

$$
\max _{x_{0}<x<x_{n}} L_{n}\left(x_{0}, \ldots, x_{n} ; x\right) \leq \max _{-1 \leq x \leq 1} L_{n}\left(x_{0}, \ldots, x_{n} ; x\right),
$$

it follows that the Lebesgue constant $\Lambda_{n}(\hat{T})$ is equal to the second local maximum $\lambda_{1}(T)$ (or $\left.\lambda_{n}(T)\right)[13,16,17]$. By using this characterization, an asymptotic expression for the 
Lebesgue constant for the extended Chebyshev nodes is given by [10]

$$
\begin{aligned}
& \Lambda_{n}(\hat{T})=\frac{2}{\pi}\left(\log (n+1)+\gamma+\log \frac{8}{\pi}\right)-\frac{4}{3 \pi}+\beta_{n}, \\
& 0<\beta_{n}<\frac{0.01}{\log ((n+1) / 4)}, n \geq 4 .
\end{aligned}
$$

Hence, we can derive the upper and lower bounds

$$
\frac{2}{\pi} \log (n+1)+0.5381 \ldots<\Lambda_{n}(\hat{T})<\frac{2}{\pi} \log (n+1)+0.5829 \ldots, \quad n \geq 4 .
$$

Also, an asymptotic expansion of $\beta_{n}$ (with unknown explicit general formula for the series coefficients, in contrast to the Chebysev nodes) can be found in [17].

As for the maximum deviation $\mathcal{M}_{n}(\hat{T})-m_{n}(\hat{T})$ of the extended Chebyshev nodes, the following estimate is given [10]:

$$
\delta_{n}(\hat{T})=\mathcal{M}_{n}(\hat{T})-m_{n}(\hat{T}) \leq 0.0196, \quad n \geq 70 .
$$

From (15) together with (14) it follows that this maximum deviation converges to

$$
\lim _{n \rightarrow \infty} \delta_{n}(\hat{T})=0.016858 \ldots
$$

\subsection{Chebyshev extrema}

The Chebyshev extrema $\bar{U}$ are the zeros of the polynomial $\left(1-x^{2}\right) T_{n}^{\prime}(x)$ and are given in explicit form as

$$
\bar{U}=\left\{x_{j}=-\cos \left(\frac{j \pi}{n}\right), j=0,1, \ldots, n\right\} .
$$

The Lebesgue constant $\Lambda_{n}(\bar{U})$ for polynomial interpolation is $[15,18]$

$$
\Lambda_{n}(\bar{U})= \begin{cases}\Lambda_{n-1}(T), & n \text { odd, } \\ \Lambda_{n-1}(T)-\alpha_{n}, & 0<\alpha_{n}<\frac{1}{n^{2}}, n \text { even }\end{cases}
$$

It is shown in [13] that the smallest local maxima $m_{n}(\bar{U})$ are bounded (in contrast to the case of the Chebyshev nodes $T$ ) by

$$
m_{n}(\bar{U})<1.57079 \ldots
$$

Thus, as in the case of the set $E, \Lambda_{n}(\bar{U})$ and $m_{n}(\bar{U})$ are of different orders of magnitude, and the maximum deviation of the local maxima $\delta_{n}(\bar{U})$ tends to infinity logarithmically.

As was proven in [13], the local maxima of $L_{n}(\bar{U} ; x)$ increase strictly monotonically from the outside toward the midpoint of the interval $[-1,1]$. This behavior suggests that the Lebesgue function $L_{n}(\bar{U} ; x)$ achieves its maximum value on the subinterval $\left(x_{n / 2}, x_{(n+2) / 2}\right)$ (or its mirror) for even degrees and on the subinterval $\left(x_{(n-1) / 2}, x_{(n+1) / 2}\right)$ for odd degrees.

Numerical observation indicates that the location of the Lebesgue constant occurs at $x^{*}(\bar{U}) \approx \frac{\pi}{2 n}$ (or its mirror) for (large) even degrees and at $x^{*}(\bar{U})=0$ for odd degrees. 


\subsection{Chebyshev nodes of the second kind}

The Chebyshev nodes of the second kind $U$ are the zeros of the $(n+1)$ th-degree Chebyshev polynomial of the second kind

$$
U_{n+1}(x)=\frac{\sin ((n+2) \arccos (x))}{\sin (\arccos (x))}
$$

and are given in closed form by

$$
U=\left\{x_{j}=-\cos \left(\frac{(j+1) \pi}{n+2}\right), j=0,1, \ldots, n\right\} .
$$

For the Lebesgue constant, it is known that $\Lambda_{n}(U)=O(n)$ [19], pp.335-339. In [13], an exact expression for $\Lambda_{n}(U)$ is given by

$$
\Lambda_{n}(U)=n+1
$$

and a lower bound for $m_{n}(U)$ is given by

$$
\frac{2}{\pi} \log (n+1)+0.3259 \ldots<m_{n}(U) .
$$

Thus, as in the cases of the sets $E$ and $\bar{U}, \Lambda_{n}(U)$ and $m_{n}(U)$ are of different orders of magnitude. In this case, the maximum deviation of the local maxima $\delta_{n}(U)$ has a linear growth.

Note that these interpolation points can be obtained from the zeros of the polynomial $\left(1-x^{2}\right) T_{n+2}^{\prime}(x)$ by deleting the zeros \pm 1 . Thus, it follows that the Lebesgue constants are sensitive to the deletion of the endpoints.

\subsection{Fekete nodes}

Since the basic Lagrange polynomials $\ell_{i}(x)$ can be expressed with the quotient of two Vandermonde determinants, namely

$$
\ell_{i}(x)=\frac{\left|V\left(x_{0}, \ldots, x_{i-1}, x, x_{i+1}, \ldots, x_{n}\right)\right|}{\left|V\left(x_{0}, \ldots, x_{n}\right)\right|}, \quad V\left(x_{0}, \ldots, x_{i}, \ldots, x_{n}\right)=\left(\begin{array}{cccc}
1 & x_{0} & \ldots & x_{0}^{n} \\
\vdots & & \vdots & \\
1 & x_{i} & \ldots & x_{i}^{n} \\
\vdots & & \vdots & \\
1 & x_{n} & \ldots & x_{n}^{n}
\end{array}\right) \text {, }
$$

we can expect that the interpolation points maximizing the Vandermonde determinant $\left|V\left(x_{0}, \ldots, x_{n}\right)\right|$ yield a small Lebesgue constant.

This node set is given by

$$
\left(1-x^{2}\right) \frac{d Q_{n}}{d x}(x)=0
$$

or, in other words, by $x_{0}=-1, x_{n}=1$, and $x_{1}, \ldots, x_{n-1}$ the extrema of the $n$ th-order Legendre polynomial $Q_{n}(x)$ and is known as the Fekete node set. There is no explicit expression for these nodes. 
For the Lebesgue constant $\Lambda_{n}(F)$, we have $\left\|\ell_{i}(x)\right\|_{\infty} \leq 1$ for $0 \leq i \leq n$, and thus the corresponding Lebesgue constant is bounded by (at most) the dimension of the interpolation space:

$$
\Lambda_{n}(F)=\max _{-1 \leq x \leq 1} \sum_{i=0}^{n}\left|\ell_{i}(x)\right| \leq n+1 .
$$

Moreover [20], the Fekete points minimize $\max _{-1 \leq x \leq 1} \sum_{i=0}^{n}\left(\ell_{i}(x)\right)^{2}$, and for these points, $\max _{-1 \leq x \leq 1} \sum_{i=0}^{n}\left(\ell_{i}(x)\right)^{2}=1$. From this, by applying the Cauchy-Schwartz inequality,

$$
\Lambda_{n}(F) \leq \sqrt{n+1}
$$

This upper bound, however, is very pessimistic. In [21], an improved upper bound for $\Lambda_{n}(F)$ is given by

$$
\Lambda_{n}(F) \leq c \log (n+1)
$$

with undetermined positive constant $c$. In addition [22], based on numerical experiments, the estimate

$$
\Lambda_{n}(F) \leq \frac{2}{\pi} \log (n+1)+0.685
$$

was conjectured. Accordingly, this confirms the conjecture in [2] that

$$
\Lambda_{n}(\hat{T})<\Lambda_{n}(F)<\Lambda_{n}(T), \quad n \geq 3
$$

\subsection{Optimal nodes}

The set of interpolation points is said to be optimal if it minimizes the Lebesgue constant. We denote the set of optimal nodes by $X^{*}$ (or the Lebesgue-optimal point set in $[-1,1]$ ):

$$
\Lambda_{n}\left(X^{*}\right)=\min _{X} \Lambda_{n}(X)
$$

Owing to the second error formula (6) and also formula (7) (for sensitivity to perturbations in the function values), it is desirable to minimize the Lebesgue constant. However, the set of optimal nodes on the interval $[-1,1]$ is known explicitly only for degrees less than four $[23,24]$, although their characterization is known from the Bernstein-Erdös conjectures.

In 1931, Bernstein [25] conjectured that the greatest local maximum of the Lebesgue function is minimal when $L_{n}(x)$ equioscillates, that is,

$$
\lambda_{1}\left(X^{*}\right)=\lambda_{2}\left(X^{*}\right)=\cdots=\lambda_{n}\left(X^{*}\right)
$$

Later, Erdös [26, 27] added to this conjecture that there is a unique canonical set $\hat{X}^{*}$ for which the smallest local maximum achieves its maximum. This is the case where the local maximum values are equal or, in other words,

$$
m_{n}(X) \leq m_{n}\left(X^{*}\right)=\Lambda_{n}\left(X^{*}\right)=\mathcal{M}_{n}\left(X^{*}\right) \leq \mathcal{M}_{n}(X) \text { for every set } X .
$$


These conjectures were proven by Kilgore [28, 29] and by de Boor and Pinkus [30]. They showed that for degree $n$, the optimal canonical interpolation set is unique, symmetric, and that its Lebesgue function must necessarily equioscillate. By using these basic properties of the optimal nodes a numerical procedure based on a nonlinear Remez search and exchange algorithm is given to compute the optimal nodes for polynomial interpolation on $[-1,1]$ [31]. Moreover, many authors (see, e.g., [22, 32]) have investigated (near) optimal point sets (in different norms) defined by the solutions of certain optimization problems.

The first sharp estimate for the optimal Lebesgue constant is given by Vértesi [33]. By constructing the following modification of the Chebyshev nodes asymptotically optimal upper and lower bounds are given [33-35], pp.110-121. Let us denote this set by

$$
\breve{T}=\left\{x_{j}=-\frac{\cos \left(\frac{\pi}{2} \frac{(2 j+1)}{(n+1)}\right)}{\cos \left(\frac{\pi}{2(n+1)}\left(1+\frac{1}{4 \log (n+1)}\right)\right)}, j=1, \ldots, n-1, x_{n}=-x_{0}=1\right\} .
$$

The Lebesgue constant $\Lambda_{n}(\breve{T})$ satisfies

$$
\begin{aligned}
c\left(\frac{\log \log (n+1)}{\log (n+1)}\right)^{2}> & \Lambda_{n}(\breve{T})-\frac{2}{\pi}\left(\log (n+1)+\gamma+\log \frac{4}{\pi}\right) \\
& > \begin{cases}\frac{\pi}{18(n+1)^{2}}+O\left(\frac{1}{(n+1)^{4}}\right), & n \text { odd, } \\
\left.-\frac{2}{\pi(n+1)}+O\left(\frac{1}{(} n+1\right)^{2}\right), & n \text { even, }\end{cases}
\end{aligned}
$$

where $c$ is an undetermined positive constant.

An application of the Erdös inequality (16), combined with the lower bound for $m_{n}(T)$ (14) and the upper bound for $\Lambda_{n}(\breve{T})$, gives

$$
\begin{aligned}
\frac{\pi}{18(n+1)^{2}}+O\left(\frac{1}{(n+1)^{4}}\right) & <\Lambda_{n}\left(X^{*}\right)-\frac{2}{\pi}\left(\log (n+1)+\gamma+\log \frac{4}{\pi}\right) \\
& <\mathrm{c}\left(\left(\frac{\log \log (n+1)}{\log (n+1)}\right)^{2}\right) .
\end{aligned}
$$

From this we can deduce that the precise growth formulas for $\Lambda_{n}\left(X^{*}\right)$ and $\Lambda_{n}(\breve{T})$ are, respectively,

$$
\Lambda_{n}\left(X^{*}\right)=\frac{2}{\pi}\left(\log (n+1)+\gamma+\log \frac{4}{\pi}\right)+o(1)
$$

and

$$
\Lambda_{n}(\breve{T})=\frac{2}{\pi}\left(\log (n+1)+\gamma+\log \frac{4}{\pi}\right)+o(1) .
$$

Since $\Lambda_{n}(\breve{T})$ and $\Lambda_{n}\left(X^{*}\right)$ have the same asymptotic growth, we can conclude that the set $\breve{T}$ has asymptotically minimal Lebesgue constants.

At this point, some remarks are useful. From (15) we can derive a precise growth formula for $\Lambda_{n}(\hat{T})$ :

$$
\Lambda_{n}(\hat{T})=\frac{2}{\pi} \log (n+1)+0.5381+\cdots+o(1) .
$$


Table 1 The values of the maximum deviations and Lebesgue constants for sets $\breve{T}, \hat{T}$, and $X^{*}$

\begin{tabular}{|c|c|c|c|c|c|}
\hline \multirow[t]{2}{*}{$n$} & \multicolumn{2}{|l|}{ set $\breve{T}$} & \multicolumn{2}{|l|}{ set $\hat{T}$} & \multirow{2}{*}{$\frac{\text { set } X^{*}}{\Lambda_{n}\left(X^{*}\right)}$} \\
\hline & $\delta_{n}(\breve{T})$ & $\Lambda_{n}(\breve{T})$ & $\delta_{n}(\hat{T})$ & $\Lambda_{n}(\hat{T})$ & \\
\hline 10 & 0.050781 & 2.056087 & 0.019471 & 2.068744 & 2.051706 \\
\hline 20 & 0.056995 & 2.463129 & 0.019340 & 2.479193 & 2.460788 \\
\hline 40 & 0.061827 & 2.887067 & 0.018952 & 2.904441 & 2.885809 \\
\hline
\end{tabular}
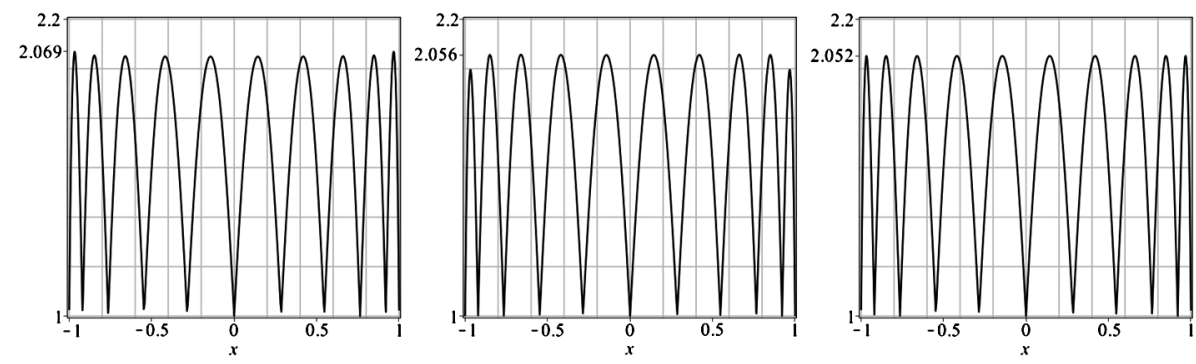

Figure 4 Graphs of $L_{10}(\hat{T} ; x), L_{10}\left(\breve{T}_{;} x\right), L_{10}\left(X^{*} ; x\right)$ from left to right.

Comparing $\Lambda_{n}(\hat{T})$ and $\Lambda_{n}(\breve{T})$, we can see that the set $\breve{T}$ is better than the set $\hat{T}$ in minimizing Lebesgue constant. Indeed, numerical results confirm this (see Table 1 and also Figure 4). The maximum deviation of the nodal set $\breve{T}$ converges to (see [34], (39))

$$
\lim _{n \rightarrow \infty} \delta_{n}(\breve{T})=0.089245 \ldots
$$

Hence, we conclude that the nodal sets studied in this section can be ordered with respect to their maximum deviation $\delta_{n}(X)=\mathcal{M}_{n}-m_{n}(X)$ and their Lebesgue constant $\Lambda_{n}(X)$ in the following way:

$$
\delta_{n}(E)>\delta_{n}(U)>\delta_{n}(\bar{U})>\delta_{n}(T)>\delta_{n}(\breve{T})>\delta_{n}(\hat{T})>\delta_{n}\left(X^{*}\right)=0
$$

and

$$
\Lambda_{n}(E)>\Lambda_{n}(U)>\Lambda_{n}(T)>\Lambda_{n}(\bar{U})>\Lambda_{n}(F)>\Lambda_{n}(\hat{T})>\Lambda_{n}(\breve{T})>\Lambda_{n}\left(X^{*}\right)
$$

\section{Concluding remarks}

In this paper we work with the interval $[-1,1]$ although all results on polynomial interpolation may be applied to any finite interval by a linear change of variable.

In view of the optimal interpolation points for the univariate polynomial interpolation, to our knowledge, both sets $\breve{T}$ and $\hat{T}$, with the position of the points given in explicit form, are the best nodal sets in the literature. Based on the Bernstein-Erdös conjecture, the nodal set $\hat{T}$ is superior than the nodal set $\breve{T}$ because of its smaller maximum deviation. When considering the optimality of a nodal set from its Lebesgue constant, the set $\breve{T}$ is better.

In the multivariate case, the problem of optimal or near optimal interpolation is much more difficult. The minimal growth of the Lebesgue constant is different for different bivariate domains. For instance, on the square, the minimal order of growth is $O\left(\ln ^{2}(n+1)\right)$, and this order is achieved for the configurations of interpolation points given in [36] and [37]. On the disk, the minimal order of growth is quite different, namely $O(\sqrt{n+1})$, as 
proved in [38]. No configurations of interpolation points obeying this order of growth are known. On the simplex, the minimal order of growth is not even known.

\section{Competing interests}

The author declares that he has no competing interests.

\section{Acknowledgements}

The author expresses his sincere thanks to Professor Annie Cuyt for her kind suggestions. This work was partially supported by the Scientific and Technical Research Council of Turkey (TUBITAK) under Grant No. 2214/B.

Received: 7 July 2015 Accepted: 20 February 2016 Published online: 12 March 2016

\section{References}

1. Davis, PJ: Interpolation and Approximation. Blaisdell, New York (1963)

2. Luttmann, FW, Rivlin, TJ: Some numerical experiments in the theory of polynomial interpolation. IBM J. Res. Dev. 9, 187-191 (1965)

3. Brutman, L: Lebesgue functions for polynomial interpolation - a survey. Ann. Numer. Math. 4(1-4), 111-127 (1997)

4. Phillips, GM: Interpolation and Approximation by Polynomials. CMS Books in Mathematics [Ouvrages de Mathématiques de la SMC], vol. 14, p. xiv+312. Springer, New York (2003)

5. Schönhage, A: Fehlerfortpflanzung bei Interpolation. Numer. Math. 3, 62-71 (1961)

6. Turetskii, $\mathrm{H}$ : The bounding of polynomials prescribed at equally distributed points. Proc. Pedag. Inst. Vitebsk 3 117-121 (1940) (in Russian)

7. Mills, TM, Smith, SJ: The Lebesgue constant for Lagrange interpolation on equidistant nodes. Numer. Math. 61(1), 111-115 (1992)

8. Trefethen, LN, Weideman, JAC: Two results on polynomial interpolation in equally spaced points. J. Approx. Theory 65(3), 247-260 (1991)

9. Tietze, H: Eine Bemerkung zur Interpolation. Z. Angew. Math. Phys. 64, 74-90 (1917)

10. Günttner, R: Evaluation of Lebesgue constants. SIAM J. Numer. Anal. 17(4), 512-520 (1980)

11. Dzjadik, VK, Ivanov, WV: On asymptotics and estimates for the uniform norms of the Lagrange interpolation polynomials corresponding to the Chebyshev nodal points. Anal. Math. 9(2), 85-97 (1983)

12. Shivakumar, PN, Wong, R: Asymptotic expansion of the Lebesgue constants associated with polynomial interpolation. Math. Comput. 39(159), 195-200 (1982)

13. Brutman, L: On the Lebesgue function for polynomial interpolation. SIAM J. Numer. Anal. 15(4), 694-704 (1978)

14. Günttner, R: Note on the lower estimate of optimal Lebesgue constants. Acta Math. Hung. 65(4), 313-317 (1994)

15. Ehlich, H, Zeller, K: Auswertung der Normen von Interpolationsoperatoren. Math. Ann. 164, 105-112 (1966)

16. Powell, MJD: On the maximum errors of polynomial approximations defined by interpolation and by least squares criteria. Comput. J. 9, 404-407 (1967)

17. Günttner, R: On asymptotics for the uniform norms of the Lagrange interpolation polynomials corresponding to extended Chebyshev nodes. SIAM J. Numer. Anal. 25(2), 461-469 (1988)

18. McCabe, JH, Phillips, GM: On a certain class of Lebesque constants. Nordisk. Tidskr. Informationsbehandling (BIT) 13 434-442 (1973)

19. Szegő, G: Orthogonal Polynomials, 3rd edn., p. xiii+423. Am. Math. Soc., Providence (1967)

20. Fejér, L: Bestimmung derjenigen Abszissen eines Intervalles, für welche die Quadratsumme der Grundfunktionen der Lagrangeschen Interpolation im Intervalle ein Möglichst kleines Maximum Besitzt. Ann. Sc. Norm. Super. Pisa, Cl. Sci. 1(3), 263-276 (1932)

21. Sündermann, B: Lebesgue constants in Lagrangian interpolation at the Fekete points. Mitt. Math. Ges. Hamb. 11(2), 204-211 (1983)

22. Hesthaven, JS: From electrostatics to almost optimal nodal sets for polynomial interpolation in a simplex. SIAM J. Numer. Anal. 35(2), 655-676 (1998)

23. Rack, HJ: An example of optimal nodes for interpolation. Int. J. Math. Educ. Sci. Technol. 15(3), 355-357 (1984)

24. Rack, HJ: An example of optimal nodes for interpolation revisited. In: Advances in Applied Mathematics and Approximation Theory. Springer Proc. Math. Stat., vol. 41, pp. 117-120. Springer, New York (2013)

25. Bernstein, S: Sur la limitation des valeurs d'un polynôme $P_{n}(x)$ de degré $n$ sur tout un segment par ses valeurs en $(n+1)$ points du segment. Izv. Akad. Nauk SSSR 7, 1025-1050 (1931)

26. Erdös, P: Problems and results on the theory of interpolation. I. Acta Math. Acad. Sci. Hung. 9, 381-388 (1958)

27. Erdös, P: Some remarks on the theory of graphs. Bull. Am. Math. Soc. 53, 292-294 (1947)

28. Kilgore, TA: Optimization of the norm of the Lagrange interpolation operator. Bull. Am. Math. Soc. 83(5), 1069-1071 (1977)

29. Kilgore, TA: A characterization of the Lagrange interpolating projection with minimal Tchebycheff norm. J. Approx. Theory 24(4), 273-288 (1978)

30. De Boor, C, Pinkus, A: Proof of the conjectures of Bernstein and Erdös concerning the optimal nodes for polynomial interpolation. J. Approx. Theory 24(4), 289-303 (1978)

31. Angelos, JR, Kaufman, EH, Henry, MS, Lenker, TD: Optimal nodes for polynomial interpolation. In: Chui, CK, Schumaker, LL, Ward, JD (eds.) Approximation Theory. VI, pp. 17-20. Academic Press, New York (1989)

32. Chen, Q, Babuška, I: Approximate optimal points for polynomial interpolation of real functions in an interval and in a triangle. Comput. Methods Appl. Mech. Eng. 128(3-4), 405-417 (1995)

33. Vértesi, P: On the optimal Lebesgue constants for polynomial interpolation. Acta Math. Hung. 47(1-2), 165-178 (1986)

34. Vértesi, P: Optimal Lebesque constant for Lagrange interpolation. SIAM J. Numer. Anal. 27(5), 1322-1331 (1990)

35. Szabados, J, Vértesi, P: Interpolation of Functions. World Scientific, Teaneck (1990) 
36. Bos, L, De Marchi, S, Caliari, M, Vianello, M, Xu, Y: Bivariate Lagrange interpolation at the Padua points: the generating curve approach. J. Approx. Theory 143(1), 15-25 (2006)

37. Bos, L, De Marchi, S, Vianello, M: On the Lebesgue constant for the Xu interpolation formula. J. Approx. Theory 141(2), 134-141 (2006)

38. Sündermann, B: On projection constants of polynomial space on the unit ball in several variables. Math. Z. 188(1), 111-117 (1984)

Submit your manuscript to a SpringerOpen ${ }^{\circ}$ journal and benefit from:

- Convenient online submission

- Rigorous peer review

- Immediate publication on acceptance

- Open access: articles freely available online

- High visibility within the field

- Retaining the copyright to your article

Submit your next manuscript at $\boldsymbol{s p r i n g e r o p e n . c o m ~}$ 